\title{
Culture de Andropogon gayanus Kunth par repiquage des éclats de souches
}

\author{
Saidou OUSSEINA ${ }^{1 *}$, Dan Guimbo IRO $^{2}$ et Douma SOUMANA ${ }^{3}$ \\ ${ }^{I}$ Département des Productions Animales, Faculté d'Agronomie, Université Abdou Moumouni, Niamey/Niger, \\ B.P.: 10960, Niamey, Niger. \\ ${ }^{2}$ Département Génie Rural et Eaux et Forêts, Faculté d'Agronomie, Université Abdou Moumouni, \\ Niamey/Niger, B.P.: 10960, Niamey, Niger. \\ ${ }^{3}$ Département de Biologie, Faculté des Sciences et Techniques, Université Abdou Moumouni, Niamey/Niger. \\ *Auteur correspondant ; E-mail : ousseys@yahoo.fr ; Tél : +227964081 13/+22790381874
}

\begin{tabular}{lll}
\hline Received: 22-12-2020 & Accepted: 23-10-2021 & Published: 30-10-2021 \\
\hline
\end{tabular}

\section{RESUME}

Les espèces herbacées rendent d'énormes services écosystémiques. Cependant, peu d'études ont été consacrées à la détermination de la production de biomasse sur une espèce fourragère particulière de Poaceae. Cette étude portant sur la banque fourragère à base de l'espèce Andropogon gayanus a été conduite sur le site expérimental de la Faculté d'Agronomie de l'Université de Niamey. L'objectif est d'étudier la reprise des souches repiquées et la production en biomasse de l'espèce Andropogon gayanus pendant la saison des pluies 2018. Le matériel biologique utilisé est constitué d'éclats de souches prélevées à partir des pieds mères de la saison pluvieuse 2017. Deux périodes de repiquage ont été étudiées : la première en début de la saison pluvieuse et la seconde au milieu de la saison pluvieuse. Deux doses d'engrais NPK de 6 et $12 \mathrm{~g}$ ont été appliquées. Le délai moyen de reprise des souches repiquées est de 6 et 4 jours respectivement pour les repiquages du début et celui du milieu de saison. La reprise s'est étalée sur 20 jours pour les souches du $1^{\text {er }}$ repiquage contre 15 jours pour celles du second. La biomasse moyenne produite est de $629,67 \mathrm{~kg} / \mathrm{ha}$ pour les souches du début de la saison alors qu'elle est de $824,01 \mathrm{~kg} / \mathrm{ha}$ pour celles du 30 juillet et $291 \mathrm{~kg} / \mathrm{ha}$ pour le témoin, à la première coupe. Cette production a augmenté à la deuxième coupe où il été récolté $720,28 \mathrm{~kg} / \mathrm{ha}$ et $1110 \mathrm{~kg} / \mathrm{ha}$ respectivement pour le premier et le second traitement, et $358,26 \mathrm{~kg} / \mathrm{h}$ a pour le témoin. Ces résultats montrent que A. gayanus est une espèce qui s'adapte et se développe bien dans les conditions hydriques favorables. L'espèce peut donc être utilisée comme une banque à fourrage, surtout dans ce milieu où les ressources fourragères deviennent de plus en plus rares et de faible valeur nutritive à certaines périodes de l'année.

(C) 2021 International Formulae Group. All rights reserved.

Mots clés : Andropogon gayanus Kunth, Repiquage, Reprise, Biomasse, Production, Niger.

\section{Fodder bank based on Andropogon gayanus Kunth by transplanting fragments of stumps}

\begin{abstract}
Grass species provide enormous ecosystem services. However, few studies have attempted to determine the biomass production on a particular fodder Poaceae species. Therefore, the present study on fodder bank based on Andropogon gayanus was carried out on the experimental site of the Faculty of Agronomy at Abdou
\end{abstract}


Moumouni University of Niamey in Niger to close the knowledge gap. The main objective of the work was to study the resumption and biomass production of the Andropogon gayanus transplanted stumps during rainy season 2018. We used stumps fragments taken from the mother plant of 2017 rainy season as biological material. Two transplanting periods were studied: the first at the start of the rainy season and the second in the middle of the rainy season. A treatment was applied with two doses of nitrogen $(\mathrm{N})$, phosphorus $(\mathrm{P})$, and potassium $(\mathrm{K})$ (NPK) fertilizer (6 and $12 \mathrm{~g}$ ). Resumption time of transplanted stumps is about 6 and 4 days respectively for transplanting of the rainy season start and that of middle of season rainy. Resumption took place over 20 days for strains of first transplanting versus 15 days for those of second one. The average biomass produced is 629.67 $\mathrm{kg} / \mathrm{ha}$ for the strains at the start of the season while it is $824.01 \mathrm{~kg} / \mathrm{ha}$ for those of July 30 and $291 \mathrm{~kg} / \mathrm{ha}$ for the control, at the first cut. This production increased in the second cut where it was harvested $720.28 \mathrm{~kg} / \mathrm{ha}$ and $1110 \mathrm{~kg} / \mathrm{ha}$ respectively for the first and second treatment, and $358.26 \mathrm{~kg} / \mathrm{ha}$ for the control. These results showed that Andropogon gayanus is a species, which adapts and develops well under favorable water conditions. The species can therefore be used as a fodder bank, especially in this environment where fodder resources are becoming increasingly scarce and of low nutritional value at certain times of the year.

(C) 2021 International Formulae Group. All rights reserved.

Keywords: Andropogon gayanus Kunth, Strains, Transplanting, Production, Niamey, Niger.

\section{INTRODUCTION}

Les difficultés d'alimentation du bétail constituent le principal frein au développement de l'élevage au Niger (MEL-Niger, 2013). L'alimentation du bétail au Niger repose sur l'exploitation des parcours naturels avec des espèces fourragères de faible qualité et de quantité étroitement dépendante de la qualité de la saison des pluies (Saidou, 2012). Le développement des cultures fourragères est très irrégulier dans les régions tropicales, notamment en Afrique sub-saharienne, malgré les résultats de la recherche (Toutain et al., 2009). Les possibilités qu'offrent théoriquement les espèces cultivées sont encore loin d'être mises à profit du fait que l'intégration de ces espèces dans les systèmes de production se heurte à de sérieuses difficultés dont on peut citer les investissements et les compétitions pour la main d'œuvre (Obulbiga et KaboréZoungrana, 2007), les référentiels techniques souvent inadaptés, l'indisponibilité fréquente de semences, les contraintes d'équipement ainsi que la rentabilité économique souvent non évidente (Klein et al., 2014).

Un des problèmes à résoudre est la réhabilitation des parcours dégradés avec des techniques adéquates susceptibles non seulement d'assurer une protection et/ ou l'amélioration du sol, mais aussi de garantir une production pouvant couvrir les besoins d'entretien et de production du bétail. La graminée, Andropogon gayanus, originaire des savanes africaines où elle prolifère tout particulièrement dans les jachères (Sawadogo, 2011) s'est distinguée des autres espèces fourragères d'une part parce que les animaux et les éleveurs l'apprécient et d'autre part cette espèce vivace sahélienne est tolère les fortes chaleurs grâce à ses particularités physiologiques (photosynthèse en C4) (Hiernaux et Le Houérou, 2006). En saison pluvieuse, la plante surprend par l'abondance de sa production et son feuillage reste à l'état vert pendant une bonne partie de la saison sèche. Des auteurs comme Somé et al. (2007) reconnaissent à cette graminée des propriétés nettoyantes et améliorantes des sols cultivés. Elle est aussi introduite en qualité de culture fourragère temporaire en assolement cultural afin d'améliorer la fertilité des sols et d'assurer une meilleure intégration des activités d'élevage dans la fermette agricole. A. gayanus a la capacité de régénérer et de se multiplier de manière végétative, d'où son introduction dans de milieux divers pour des objectifs divers et multiples. L'espèce est considérée par les Peuls comme l'une des meilleures graminées pastorales pour les zébus surtout pendant la 
saison sèche. De plus, A. gayanus peut être utilisée pour la constitution de réserves sous forme de foin et supporte les fauches répétées (Obulbiga et al., 2014). Elle peut donc être utilisée dans les pâturages artificiels.

Pour améliorer le système d'alimentation du bétail et réduire le coût de l'alimentation des animaux, qui constitue un frein au développement de l'élevage de bovins au sahel, la maîtrise de la production fourragère semble être une solution alternative urgente. L'objectif de cette étude était d'évaluer la reprise des souches de A. gayanus repiquées ainsi que l'effet du NPK et de la coupe sur la production en biomasse de cette espèce pendant la saison de pluies.

\section{MATERIEL ET METHODES}

\section{Site d'étude}

L'étude a été menée au parc expérimental de la Faculté d'Agronomie de l'Université Abdou Moumouni situé dans le $5^{\mathrm{e}}$ arrondissement de la ville de Niamey. Le climat est de type sahélo-soudanien avec des températures élevées $\left(28^{\circ} \mathrm{C}\right.$ à $\left.40^{\circ} \mathrm{C}\right)$ entre avril et juin et basses $\left(13^{\circ} \mathrm{C}\right.$ à $\left.22^{\circ} \mathrm{C}\right)$ entre décembre et janvier (INS, 2017). Les données pluviométriques enregistrées à la faculté d'agronomie de 2010 à 2018 montrent un cumul variant entre 400 et $600 \mathrm{~mm}$ par an à l'exception de quelques années où il a été enregistré un cumul supérieur à $700 \mathrm{~mm}$.

\section{Matériel Biologique}

Les souches d'Andropogon gayanus utilisées provenaient des souches-mères produites à partir de semences pendant la saison pluvieuse 2017 sur une parcelle de la Faculté d'Agronomie.

\section{Dispositif Expérimental}

Le dispositif principal est composé de deux (2) séries de 6 parcelles, soit un total de 12 parcelles de dimensions $16,60 \mathrm{~m}$ x 5,80 m chacune et espacées de $2 \mathrm{~m}$. A l'intérieur de chaque parcelle, six (6) lignes parallèles distantes d'un (1) $m$ sont matérialisées dans le sens de la longueur. Des poquets de $30 \mathrm{~cm}$ de diamètre et $30 \mathrm{~cm}$ de profondeur ont été creusés sur les lignes avec un écartement inter-poquets de $60 \mathrm{~cm}$. Une bordure de $50 \mathrm{~cm}$ est laissée sur chaque côté de la parcelle expérimentale. Après la reprise des souches, des apports d'engrais NPK ont été réalisés selon le schéma suivant :

- trois premiers poquets d'une ligne reçoivent une dose de $6 \mathrm{~g}$ de NPK chacun et constituent le traitement $\mathrm{T} 1$;

- trois poquets suivants avec une dose de $12 \mathrm{~g}$ de NPK chacun représentent le traitement T2 ;

- trois autres poquets suivants laissées sans traitement d'engrais, constituent le témoin T0, et ainsi de suite jusqu'à la fin de la ligne.

\section{Mise en place des souches}

Le repiquage des souches a été effectué sur deux périodes. Le premier a lieu le 2 juillet (début de la saison pluvieuse) (série 1) et le second le 30 juillet (milieu de la saison pluvieuse) (série 2). Cinq (5) jours avant les prélèvements des souches, les souches mères ont été fauchées à $15 \mathrm{~cm}$ du sol afin de réduire la perte d'eau par évapotranspiration chez les souches repiquées. La mise en place des souches a été effectuée le jour du prélèvement.

Modalités de réalisation du repiquage des souches

Au total 1296 poquets ont été réalisés, soit 648 poquets par série, au cours des 2 périodes de repiquage. Des observations quotidiennes ont porté sur l'évolution de la reprise.

\section{Évaluation de la biomasse aérienne}

L'estimation de biomasse aérienne, sous l'effet de l'apport de deux doses d'engrais de NPK (15-15-15) une semaine après le repiquage, a été réalisée sur les parcelles de la série 1. La première récolte a eu lieu lorsque les souches ont atteint $40 \mathrm{~cm}$ de hauteur après repiquage. La seconde est intervenue sur les mêmes souches lorsqu'elles ont atteint la même hauteur. La récolte a consisté à couper toutes les talles du poquet à partir de $5 \mathrm{~cm} \mathrm{du}$ 
collet. Le séchage de la biomasse a été effectué à l'étuve à $70^{\circ} \mathrm{C}$ jusqu'à l'obtention d'un poids constant.

\section{Traitement des données et expression des résultats}

Les données recueillies ont permis de calculer les paramètres suivants :

- Le délai de reprise (en jours) : temps qui sépare la mise en terre des souches et la reprise de la première souche ;

- La durée de reprise des souches ou temps qui s'écoule entre la reprise de la première souche et celle de la dernière souche ;

- Le taux de reprise de souches repiquées qui est le rapport entre le nombre de souches ayant repris et le nombre total des souches repiquées ;

- Le taux de survie des souches: qui correspond au rapport entre le nombre de talles réussis par souche ayant repris et le nombre total de talles par souche repiquée ;

- La production de biomasse épigée sous l'effet des traitements $\mathrm{T} 1$ et $\mathrm{T} 2$.

Les moyennes associées au coefficient de variation des différents paramètres ont été calculées. Ces moyennes ont ensuite été comparées par un test d'ANOVA le logiciel SPSS Statistics 20. Le seuil de probabilité de 0,05 en dessous duquel l'hypothèse nulle (égalité entre les moyennes) est rejetée a été retenu.

\section{RESULTATS \\ Reprise des souches}

Les paramètres de reprise des souches diffèrent d'une série à une autre comme le montre le Tableau 1. Toutefois, les souches repiquées ont présenté un début de flétrissement pendant les premiers jours qui ont suivi la transplantation.

Les souches repiquées au début de la saison pluvieuse ont un taux de reprise inférieur à celui des souches repiquées au milieu de la saison avec respectivement $76,91 \%$ et $88,25 \%$. Le délai moyen de reprise de 4 jours observé pour la $2^{\text {ème }}$ période de repiquage est plus court que celui de la $1^{\text {ère }}$ période qui est de 6 jours. La survie des souches est nettement plus faible pour les souches repiquées au début de la saison pluvieuse, avec un taux de $46,91 \%$, alors que celles repiquées au milieu de la saison pluvieuse ont un taux de $80,04 \%$.

Pour tous les paramètres de reprise des souches étudiés, celles repiquées pendant l'installation effective de la saison pluvieuse ont mieux supporté le repiquage.

\section{Production de la biomasse aérienne}

La production de biomasse a significativement varié entre la première coupe et la seconde d'une part et entre les traitements d'autre part (Figure 1). Ainsi, la dose d'engrais a eu un effet significatif sur la production de biomasse aérienne. En effet, à la première récolte, la production en biomasse épigée de 824,01 kg.MS/ha avec la dose de $12 \mathrm{~g}$ de NPK est significativement plus élevée $(\mathrm{P}=0,038)$ que celle obtenue avec la dose de 6 g $(629,67$ kg.MS/ha). Il en est de même pour la deuxième récolte aux doses de 12 et $6 \mathrm{~g}$ d'engrais, avec respectivement une production de 1110 kg.MS/ha et $720,28 \mathrm{~kg} . \mathrm{MS} / \mathrm{ha}(\mathrm{P}=0,04) . \mathrm{La}$ production est statistiquement plus importante à la deuxième coupe pour tous les traitements. Les souches fertilisées présentent une production moyenne en biomasse supérieure à celle des témoins (souches non fertilisées), qui passe du simple chez le témoin (291 kg.MS/ha et $358,26 \mathrm{~kg} . \mathrm{MS} / \mathrm{ha}$ respectivement à la $1^{\text {ère }}$ et à la $2^{\text {ème }}$ coupe) au double avec la dose de $6 \mathrm{~g}$ de NPK et au triple pour la dose de $12 \mathrm{~g}$. Pour la $2^{\text {ème }}$ coupe, les souches ont atteint la hauteur de $40 \mathrm{~cm}$ au $10^{\text {ème }}$ jour après la première, soit une croissance moyenne de $4 \mathrm{~cm}$ par jour. 
Tableau 1: Paramètres de reprise des souches.

\section{Périodes de repiquage}

\section{Paramètres}

Série 1

Série 2

début saison pluvieuse milieu saison pluvieuse

\begin{tabular}{lcc}
\hline Délais de reprise (jours) & 6 & 4 \\
\hline Durée de la reprise (jours) & 20 & 15 \\
\hline Taux de reprise (\%) & 76,91 & 88,25 \\
\hline Taux de survie des souches (\%) & 46,91 & 80,04 \\
\hline
\end{tabular}

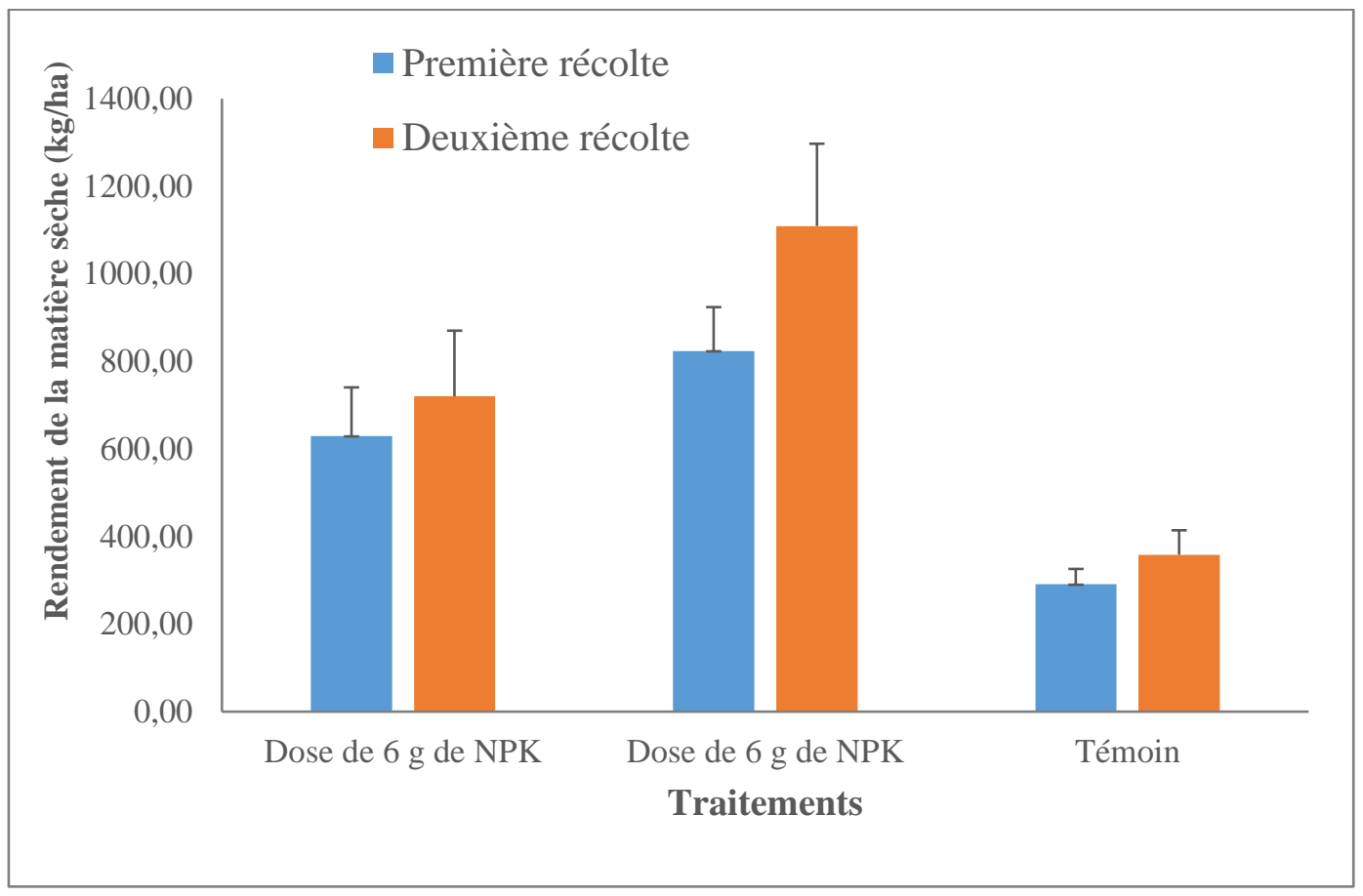

Figure 1 : Production de la biomasse sèche des souches de Andropogon gayanus fertilisées avec de NPK.

\section{DISCUSSION}

La transplantation d'éclats des souches de Andropogon gayanus a été suivie d'un flétrissement des feuilles et des tiges sur les deux types d'essai. Il s'agit d'un traumatisme physiologique qui s'est traduit par un assèchement des tiges et des feuilles avant leur reprise. Cette situation peut être due à une détérioration du système racinaire qui par la suite a entrainé un flétrissement des tiges et des feuilles. Le faible taux de survie des souches repiquées obtenu au début de la saison pluvieuse (2 juillet) traduit une phase d'adaptation ou une mauvaise implantation de l'espèce pendant cette période. Cette situation pourrait s'expliquer par un taux d'humidité du sol qui est faible et aux pluies très espacées du début de saison. Ceci rend les souches 
vulnérables aux attaques des termites qui d'abord s'intéressent aux tiges déshydratées comme l'ont déjà souligné Zougmoré et al. (2000). Ces mêmes auteurs ont affirmé qu'en cas de sècheresse, des attaques des termites peuvent être fréquentes et avoir des répercussions sur le tallage et la croissance des plantules et par conséquent sur la production de biomasse. Toutefois, Mitja et De Robert (2004) ont rapporté une bonne tolérance de cette espèce à la sécheresse, due certainement à sa capacité de s'enraciner profondément et de puiser dans les réserves hydriques du sol. Klein et al. (2014) ajoutent que A. gayanus est résistant aux attaques parasitaires même les plus redoutables comme l'ont déjà rapporté Mitja et De Robert (2004). Les taux moyens de survie des souches de A. gayanus relevés au cours des deux périodes de repiquage indiquent que l'espèce s'adapte et s'implante bien par repiquage comme l'a également souligné Tassembédo (2001). Ceci laisse présager que la période de repiquage pourrait expliquer la différence observée entre les taux de reprise des souches (César et al., 2004) et que la saison pluvieuse est bien favorable au repiquage. En effet, le taux de reprise varie au cours de la saison pluvieuse. Dans le cas de cette étude, la reprise est faible en début de saison de pluies et devient plus importante avec l'installation effective de la saison des pluies. Ces résultats corroborent ceux obtenus par Buldgen et Dieng (1997) qui affirment que la réussite du repiquage dépend du choix et de la préparation de talles, de l'absence d'attaque parasitaire et surtout de l'alimentation optimale en eau des talles repiquées. Zougmoré et al. (2000) affirment également que des résultats similaires (taux de reprise inférieurs à 60\%) peuvent être obtenus lorsque la pluviosité n'est pas régulière ou lorsque la vigueur de brins mère reste faible.

La différence significative entre le rendement de biomasse selon la dose de NPK peut être due à un effet favorable suite à l'apport des éléments nutritifs. Ce résultat est confirmé par Obulbiga (2002) qui a rapporté que la production de la matière sèche la plus élevée est obtenue avec la dose maximale de fumure azotée. Toutefois, Mitja et De Robert
(2004) affirment que A. gayanus produit une forte biomasse pour nourrir le bétail, sur des sols peu fertiles et sans apport d'engrais. Obulbiga (2002) rapporte, quant à lui, que le rythme de coupe a une influence très significative sur la production de la matière sèche. Par contre, ce résultat contredit celui de Cissé et Breman (1980) qui ont montré que la coupe a tendance à avoir un effet négatif sur la production de biomasse en milieu naturel. Dans le cas du présent travail, cette production a augmenté avec la coupe chez tous les traitements et le lot témoin. Cette différence pourrait être expliquée par l'effet "engrais" dans cette étude. La production de biomasse obtenue est largement inférieure à celle obtenue par Obulbiga et al. (2014) qui est de $3434 \mathrm{~kg} . \mathrm{MS} / \mathrm{ha}$ et $3500 \mathrm{~kg} . \mathrm{MS} / \mathrm{ha}$ respectivement pour des coupes à 40 jours et à 30 jours après le repiquage. Cette différence peut être due au fait que, dans la présente étude la coupe est faite en fonction de la hauteur (40 $\mathrm{cm}$ ) des plants et non en fonction du nombre de jours. De même, dans le cas de cette étude, les coupes sont faites au moment où les plants sont jeunes et le rapport feuilles/tiges plus grand, ce qui permet une meilleure utilisation fourragère de A. gayanus (Obulbiga, 2002). Toutefois, les résultats $(1110 \mathrm{~kg} . \mathrm{MS} / \mathrm{ha})$ s'approchent de ceux de Ahmat (2008) qui a obtenu 1,88 tonnes /ha au Tchad et en apportant plus de fertilisant organique et minéral. A. gayanus a donc tendance à se développer convenablement dans le milieu au cours des premières années qui suivent sa plantation (Mitja et De Robert 2004).

\section{Conclusion}

La présente étude a montré que l'espèce A. gayanus s'adapte et s'implante bien par repiquage de souches lorsque les conditions hydriques sont favorables. La reprise des éclats de souches est meilleure avec l'installation effective de la saison pluvieuse et la régularité des pluies dans le temps. Le rendement en biomasse est acceptable lorsque la plante a atteint $40 \mathrm{~cm}$ de hauteur et le fourrage, comportant moins de tiges, peut être utilisée valablement en alimentation animale. Aussi, il serait intéressant de poursuivre l'étude, en 
intégrant les aspects qualitatifs du fourrage (composition chimique, valeur nutritive, productions permises) et les effets du broutage sur la plante.

\section{CONFLIT D'INTERETS}

Les auteurs déclarent qu'ils n'ont aucun conflit d'intérêts.

\section{CONTRIBUTIONS DES AUTEURS}

Dans le cadre de ce travail, OS a dirigé l'élaboration du protocole de recherche, le traitement des données et la rédaction du manuscrit. IDG a contribué régulièrement à l'élaboration du manuscrit dans le but de l'améliorer et SD a formulé des propositions importantes au déroulement de ce travail.

\section{REFERENCES}

Ahmat H. 2008. Contribution à la promotion des cultures fourragères en élevage traditionnel : Réalisations du PDR/B-Tch 50 dans la sous-préfecture de Djédaa au Tchad. Mémoire de diplôme d'études approfondies de productions animales, EISMV, Dakar. 32 p.

Buldgen A, Dieng A. 1997. Présentation générale d'Andropogon gayanus Kunth var. Bisquamulatus (Hochst,) Hack. In Andropogon gayanus var. Bisquamulatus. Une culture fourragère pour les régions tropicales. Presses Universitaires de Gembloux : Gembloux, Belgique ; $171 \mathrm{p}$.

César J, Ehouinsou M, Gouro A. 2004. Conseils et formation en appui à la production laitière: Production fourragère en zone tropicale et conseils aux éleveurs, CIRAD- EMVT- France, 48p.

Cissé M, Breman H. 1980. Influence de l'exploitation sur un pâturage à Andropogon gayanus Kunth var. tridentatus. Rev. Elev. Méd. Vét. Pays Trop., 33 (4) : 407-416.

Hiernaux P, Le Houérou H-N. 2006. Les parcours du Sahel. Sécheresse, 17 (1-2) : $1-21$.

INS-Niger. 2017. Annuaire statistique 20132017. Edition 2018. 260 p.
Klein HD, Rippstein G, Huguenin J, Toutain B, Guérin H, Louppe D. 2014. Les Cultures Fourragères. Ed. Quae : Versailles ; 262 p. (Agricultures tropicales en poche) DOI : https://doi.org/10.35690/978-27592-2169-1

MEL-Niger (Ministère de l'Elevage Niger). 2013. Stratégie de développement durable de l'élevage (SDDEL 203-2035). $83 \mathrm{p}$.

Mitja D. 1992. Influence de la culture itinérante sur la végétation d'une savane humide de Côte-d'Ivoire (Booro-Borotou-Touba). Coll. «Etudes et Thèses », ORSTOM, Paris, $241 \mathrm{p}$.

Mitja D, Leal Filho N, Topall O. 1998. Pour une réhabilitation des pâturages amazoniens dégradés, l'exemple de andropogon gayanus kunth, (Marabâ, Parâ, Brésil). Rev. Eco/. (Terre Vie), $\mathbf{5 3}$ : 39-57.

Mitja D, De Robert P. 2004 : Des agriculteurs innovateurs : une nouvelle graminée dans les pâturages de Santa Maria (Amazonie brésilienne). Natures, Sciences, Sociétés, 12 : 285-298.

Obulbiga MF. 2002. Influence de la fumure azotée et du rythme d'exploitation sur la production d'Andropogon gayanusKunth cultivée en zone nord soudanienne du Burkina Faso. Mémoire de diplôme d'Etude Approfondie. Université polytechnique de Bobo Dioulasso, IDR.67p.

Obulbiga MF, Bougouma V, Sanon HO, Gnanda I. 2014. Production à double objectif d'andropogon gayanuskunth, en culture pluviale dans le plateau central du Burkina Faso. Agronomie Africaine, 26 (3) : 289-298.

Obulbiga MF, Kaboré-Zoungrana CY. 2007. Influence de la fumure azotée et du rythme d'exploitation sur la production de matière sèche et la valeur alimentaire de Andropogon gayanus Kunth au Burkina Faso. TROPICULTURA, 25 (3) : 161-167.

Sawadogo I. 2011 : Ressources fourragères et représentations des éleveurs, évolution des pratiques pastorales en contexte 
d'aire protégée: Cas du terroir de Kotchari à la périphérie de la réserve de biosphère du $\mathrm{W}$ au Burkina Faso. Environnement et Société. Museum national d'histoire naturelle - MNHN PARIS, 2011. Français. https://tel.archives-ouvertes.fr/tel00708327

Saidou O. 2012. Caractérisation des ressources fourragères de la Station Sahélienne Expérimentale de Toukounous (FilinguéNiger). Thèse de Doctorat de l'Université Abdou Moumouni de Niamey, Niger, Faculté d'Agronomie, $135 \mathrm{p}$.

Some AN, Traoré K, Traoré O, Tassambedo M. 2007. Potentiel des jachères artificielles à Andropogon spp. dans l'amélioration des propriétés chimiques et biologiques des sols en zone soudanienne (Burkina Faso).
$B A S E$ [En ligne], 11(3) : 245-252. URL : https://popups.uliege.be/1780-

4507/index.php?id=892.

Tassambédo MA. 2001. Amélioration de la fertilité des sols sous couverture à Andropogon spp. : Effets sur le raccourcissement de la jachère sur un sol ferrugineux tropical et sur l'augmentation de la production (Zone soudanienne du Burkina Faso). Rapport de stage de fin de 1ère année IDR, option Eaux et Forêts, 49 p.

Zougmoré R, Thiombiano L, Kambou F, Zida Z. 2000. Végétalisation de cordons pierreux au moyen du vétiver ou de l'andropogon. Fiche technique $\mathrm{n}^{\circ} 2$, INERA CRREA-CENTRE Saria, Koudougou, Burkina Faso. 3p. 